\title{
Interview Bernd Fitzenberger für „Wirtschafts- und Sozialstatistisches Archiv“6
}

\author{
Walter Krämer
}

Online publiziert: 18. Februar 2022

(C) Springer-Verlag GmbH Germany, part of Springer Nature 2022

Bernd Fitzenberger (* 14. Mai 1963 in Friedberg) hat in Konstanz Volkswirtschaftslehre und Mathematik (Diplom in VWL 1987, Vordiplom in Mathematik 1989) und an der Universität Stanford Statistik (Master 1992) studiert, 1993 in Stanford in Ökonometrie promoviert und sich 1998 in Konstanz mit einer Schrift in der empirischen Arbeitsmarktforschung habilitiert. Im gleichen Jahr wurde er als Professor für Volkswirtschaftslehre, insbesondere Sozialpolitik an die TU Dresden berufen. Ein Jahr später wechselte er auf einen Lehrstuhl Volkswirtschaftslehre, insbesondere Ökonometrie an der Universität Mannheim, von dort im Jahre 2004 auf einen Lehrstuhl für Volkswirtschaftslehre, insbesondere Labor Economics an der Goethe-Universität Frankfurt, im Jahr 2007 auf einen Lehrstuhl für Statistik und Ökonometrie an der Universität Freiburg und im April 2015 auf einen Lehrstuhl für Ökonometrie an der Humboldt-Universität zu Berlin. Ihr vorläufiges Ende fand diese beeindruckende Laufbahn mit der Übernahme des Direktorenpostens des Instituts für Arbeitsmarkt- und Berufsforschung im September 2019, verbunden mit einer Professur für Quantitative Arbeitsökonomik an der Universität Erlangen Nürnberg.

Bernd Fitzenberger ist Research Fellow am Forschungsinstitut zur Zukunft der Arbeit (IZA) in Bonn, am CESifo in München und am Research Center for Education and the Labour Market in Maastricht, sowie International Research Affiliate am Institute for Fiscal Studies in London. Des Weiteren ist er Co-Editor der internationalen Zeitschrift Labour Economics, Associate Editor der internationalen Zeitschrift Empirical Economics und Mitglied im Herausgebergremium des Journals for Labour Market Research. Er koordinierte verantwortlich das von 2014 bis 2022 laufende DFG-Forschungsschwerpunktprogramm ,The German Labor Market in a Globalized World: Challenges through Trade, Technology and Demographics“ (SPP 1764)

Walter Krämer $(\square)$

Universität Dortmund, Dortmund, Deutschland

E-Mail: walterk@statistik.uni-dortmund.de 
Abb. 1 Bernd Fitzenberger $2021 \mathrm{im} \mathrm{IAB}$

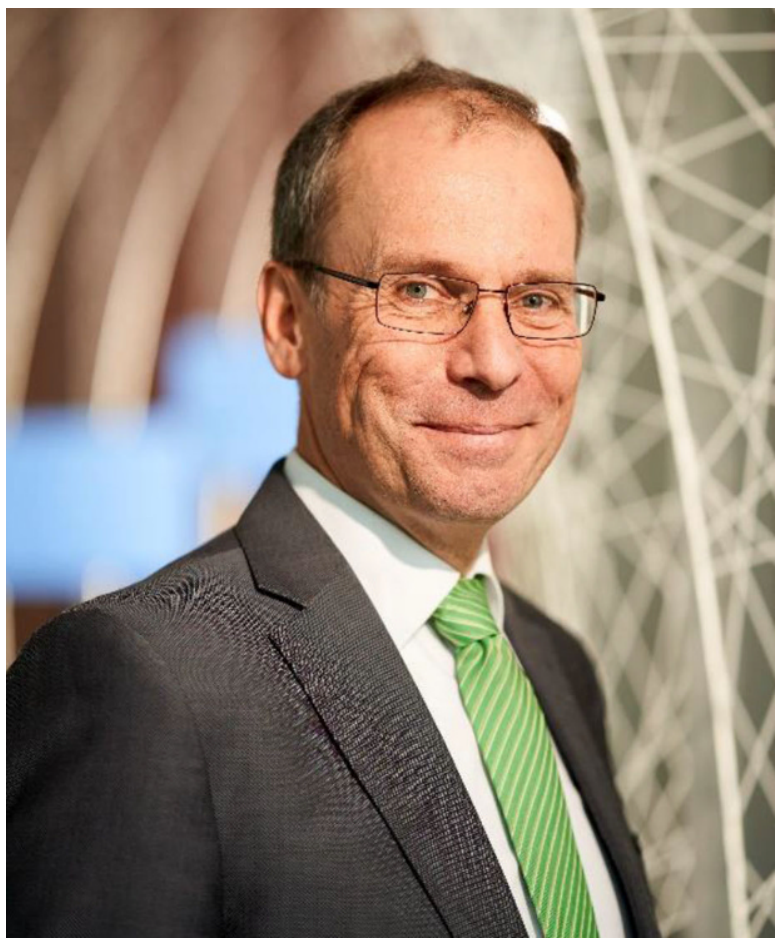

und ist Mitglied der Leopoldina (Sektion Ökonomik und Empirische Sozialwissenschaften). Im Jahr 2020 erhielt er den Deutschen Wirtschaftspreis der Joachim Herz Stiftung für das beste Forschungswerk eines etablierten Wissenschaftlers zur Ökonomik der Arbeit.

Bernd Fitzenbergers Hauptforschungsgebiete sind Einkommens- und Lohnungleichheit, Beschäftigungsentwicklung, Evaluation von Maßnahmen der Arbeitsmarkt- und Bildungspolitik, berufliche Bildung und Übergang von Schule zu Beruf, Beschäftigung von Müttern, Gewerkschaften (Tarifbindung, Organisationsgrad, Lohnstrukturen und Beschäftigung), Evaluationsmethoden und Methoden der Quantilsregression. Die Mitglieder der Deutschen Statistischen Gesellschaft kennen ihn auch als Vertreter der Statistik und Ökonometrie im Fachkollegium Wirtschaftswissenschaften der Deutschen Forschungsgemeinschaft. Die Fragen stellte Walter Krämer. (Abb. 1).

Herr Fitzenberger, Sie haben Ihren Doktortitel in den USA erworben und dazu auch noch an einem der berühmtesten Plätze überhaupt. Was können wir diesbezüglich von den Amerikanern lernen?

Einiges. In Stanford - ein phantastischer Ort, der mich sehr geprägt hat - konnte ich Geistesgrößen wie Karl Popper oder Spitzen meiner Fächer VWL, Ökonometrie und Statistik persönlich erleben, wie Ken Arrow, James Tobin, Jim Heckman, Clive Granger, Takeshi Amemiya, T.W. Anderson oder Bradley Efron, auch angehen- 
de weibliche Stars wie Penny Goldberg, Hilary Hoynes oder Susan Athey, oder Menschen der Zeitgeschichte wie Michail Gorbatschow und Helmut Schmidt. Eine solche intellektuelle Konzentration und Offenheit erlebbar zu machen, da machen uns die amerikanischen Spitzenuniversitäten einiges vor.

\section{Und betreffend die Ausbildung?}

Auch eine Menge. Als Diplom-Volkswirt mit einem Vordiplom in Mathematik war ich sehr gut für eine Promotion vorbereitet, habe aber trotzdem im PhD-Programm in Stanford noch extrem viel gelernt. Diese Programme bereiten in einem weit umfassenderen Maß auf eine forschungsorientierte Promotion vor, als das damals in Deutschland der Fall gewesen ist. Mit den neuen Doktorandenprogrammen in der VWL haben wir inzwischen zwar wichtige Schritte in diese Richtung getan, sind aber nach meiner Einschätzung noch weit entfernt von der breiten Exzellenz der Ausbildung, wie ich sie selbst in Stanford erlebt habe.

Beeindruckt war ich auch von der Unkompliziertheit und Selbstverständlichkeit, mit der ich als Nichtamerikaner in einem internationalen Jahrgang mit einem Ausländeranteil von ca. $50 \%$ in das Doktorandenprogramm aufgenommen und von der Universität ab dem zweiten Jahr ohne Vorfestlegung auf einen Betreuer finanziert wurde. Als ich nach Stanford ging, wollte ich Zeitreihenökonometriker und empirisch arbeitender Makroökonom mit dem Fokus auf Arbeitsmärkte werden. Promoviert habe ich bei einem mikroökonometrisch versierten Arbeitsökonomen zu einem methodischen Thema an der Schnittstelle zwischen Zeitreihenökonometrie und Mikroökonometrie. Diese methodischen Kenntnisse habe ich als empirischer Arbeitsmarktforscher später vielfach angewandt (siehe etwa Fitzenberger 1999; Fitzenberger et al. 2001 oder Fitzenberger et al. 2004) - aber prägend war vor allem die Erfahrung, dass die Mikroökonometrie in Verbindung mit tollen Mikrodaten der Schlüssel zum Erkenntnisgewinn in der empirischen Arbeitsmarktforschung war und immer noch ist. So selbstverständlich dies für das Fach auch in Deutschland heute ist, so revolutionär war das für mich damals in den USA.

Und der Anfang Ihres Wegs dahin? War das Ziel Statistik geplant oder zufällig?

Durchaus geplant, mit einigen glücklichen Zufällen. Im Mathe-Leistungskurs in der Schule hat mir im zweiten Halbjahr der 13. Klasse, als das Abitur schon gelaufen war, der Statistikschwerpunkt am meisten Spaß gemacht. Und danach spielte Statistik in meinem Studium in Konstanz eine große Rolle - schließlich studierte ich in der damaligen Fakultät für Wirtschaftswissenschaften und Statistik. Da war ich auch schon früh als Tutor in die Statistikausbildung im Grundstudium eingebunden, dadurch habe ich extrem viel gelernt. Und durch Gerd Ronning erhielt ich erste Einblicke in die Mikroökonometrie - und in eine Vorabversion seines späteren Lehrbuchs zu diesem Thema (Ronning 1991). Und im vierten Jahr meines VWL-Studiums habe ich begonnen, Mathematik bis zum Vordiplom zu studieren, um u. a. die Methoden in Statistik und Ökonometrie wirklich zu verstehen. Das hat mir in Stanford sehr geholfen. (Abb. 2). 
Abb. 2 Bernd Fitzenberger (rechts) mit dem schweizerischen Ökonomen Antonio Manzini (links) bei der Abschlussfeier zur Verleihung des Master of Science in Statistics im Juni 1992 an der Stanford University

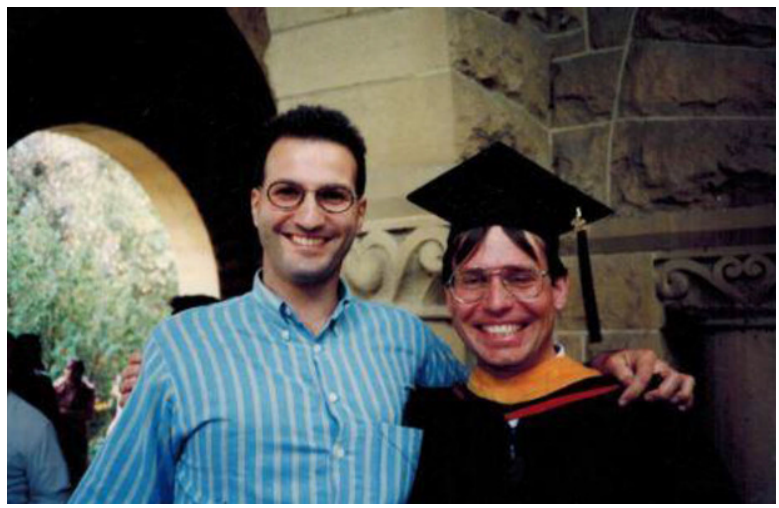

Wo Sie dann eine eher methodische Dissertation geschrieben haben?

Ja, deren Kern wurde dann später im Journal of Econometrics publiziert (Fitzenberger 1998). Ausschlaggebend dafür war Takeshi Amemiya; er hat mir geraten, auch Kurse im PhD-Programm in Statistik zu belegen und über diesen Weg einen Master in Statistics unter Anrechnung meiner im VWL-Programm absolvierten Ökonometriekurse zu machen. Dieser Rat war goldrichtig - und so hatte ich mit meiner Promotion auch gleich einen MSc in Statistics. Das ist ein solides Fundament, auf dem ich mich über meine Karriere methodisch immer wieder weiterentwickeln konnte, obwohl ich nach der Promotion eine angewandte Ausrichtung als empirischer Arbeitsmarktforscher eingeschlagen habe ...

... die Sie dann nach Nürnberg an die Spitze des Instituts für Arbeitsmarkt- und Berufsforschung geführt hat. Wie ordnet sich denn das IAB in den Chor der deutschen Sozial- und Wirtschaftsforschungsinstitute ein?

Das IAB betreibt Arbeitsmarkt-, Berufs- und Sozialpolitikforschung im Rahmen eines im Sozialgesetzbuch II und III festgeschriebenen Auftrags. Es ist eine Dienststelle der Bundesagentur für Arbeit (BA) und berät die BA und das Bundesministerium für Arbeit und Soziales im Rahmen seines gesetzlichen Auftrags. Soweit ist es einem Ressortforschungsinstitut vergleichbar. Gleichzeitig sind das IAB und seine Forscherinnen und Forscher aber wissenschaftlich unabhängig und neben der Forschung auch in Datenproduktion und evidenzbasierter Politikberatung tätig. Neben eigenständigen Datenerhebungen kann das IAB als ein Alleinstellungsmerkmal durch seinen Zugang zu den Prozessdaten der BA einzigartige Datenbestände für die Forschung erschließen. Diese sind dann über das Forschungsdatenzentrum der BA im IAB (www.fdz.iab.de) für die externe Wissenschaft in datenschutzkonformer Weise zugänglich. Als weiteres Alleinstellungsmerkmal hat das IAB durch die Einbindung in die BA auch die Möglichkeit, Feldexperimente im Bereich der Arbeitsmarktpolitik durchzuführen.

Wegen seiner thematischen Festlegung und seines gesetzlichen Beratungsauftrags ist das IAB mit den Leibniz-Instituten in den Wirtschafts- und Sozialwissenschaften nicht vergleichbar. Aber seine wissenschaftliche Freiheit, seine exzellenten Forschungserfolge, wie zuletzt durch die Begutachtung durch den Wissenschaftsrat 
Abb. 3 Bernd Fitzenberger (Mitte) beim internationalen Workshop ,Vacancies, Hiring and Matching“ am 1./2. Oktober 2019 anlässlich des 30-jährigen Bestehens der IAB-Stellenerhebung. Links Josef Zweimüller, rechts Nicole Gürtzgen

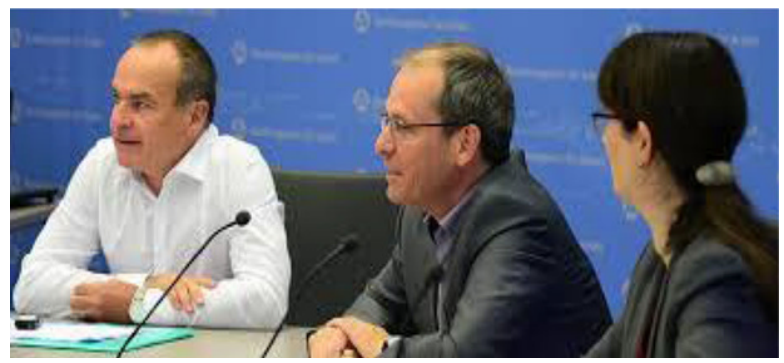

2019 bestätigt, die Tatsache, dass das IAB ein englischsprachiges, interdisziplinäres Doktorandenprogramm (GradAB) zusammen mit der WiSo-Fakultät an der Friedrich-Alexander Universität Erlangen-Nürnberg betreibt, seine intensiven Forschungskooperationen und seine Drittmittelerfolge weisen das IAB als exzellente wissenschaftliche Forschungseinrichtung aus.

Haben Sie als Chef dieser Organisation, der wohl jedes Jahr Dutzende von Akademikern neu anstellt, den Eindruck, dass die Statistikausbildung in Deutschland gut funktioniert?

Teils-teils. Einerseits hat es in den letzten Jahrzehnten in der statistischen Methodenausbildung in der Soziologie und in der Volkswirtschaftslehre große Fortschritte gegeben. Unser Nachwuchs bringt dementsprechend einen tollen Werkzeugkasten und Erfahrungsschatz in der Anwendung moderner statistischer Methoden mit. Bei Studierenden der Wirtschaftswissenschaften fehlen jedoch mehr als früher profunde Kenntnisse in der Wirtschaftsstatistik und der amtlichen Statistik. In meiner Zeit als Habilitand haben mir mein Lehrer Wolfgang Franz und mein Mentor Reinhard Hujer, beide in der Forschung und Politikberatung sehr aktiv und erfolgreich, die Wichtigkeit von Kenntnissen in Wirtschaftsstatistik überzeugend deutlich gemacht. Diese Kenntnisse sind im IAB - und auch in der BA, die Teil der amtlichen Statistik ist - ganz zentral.

Die Statistik an den Hochschulen steht jedoch vor einem Dilemma - sie ist verstreut über viele Disziplinen. Gleichzeitig ist es jedoch für die Entwicklung und Verbreitung neuerer Methoden wichtig, dass das Fach in seiner ganzen Breite zusammenkommt und zusammenwirkt, um über den Tellerrand der jeweiligen Anwendungsdisziplin hinauszusehen. Hier kommt den Statistikstudiengängen - bspw. in Dortmund, München oder auch in Berlin, wo ich mehrere Jahre die Koordination des Masters in Statistik innehatte - und der Fakultät/der Fachgruppe in Statistik in Dortmund und München eine sehr wichtige Rolle zu. (Abb. 3).

Das wird meine Kollegen und Kolleginnen in Dortmund freuen. Was sollte man denn Ihrer Auffassung nach da anders machen?

Für das skizzierte Dilemma gibt es keine einfachen Lösungen. Einerseits ist es wichtig, dass sich die Statistik als eigenständiges Fach definiert und die Möglichkeiten nutzt, Fachgruppen oder Fakultäten mit eigenen Studiengängen zu bilden. In Zeiten von Data Science und der Erschließung neuer Daten können hierfür Gele- 
genheitsfenster entstehen. Andererseits ist die enge Kooperation mit den Anwendungswissenschaften extrem wichtig - und statistische Grundkompetenzen sind in vielen Studiengängen eine berufsqualifizierende Schlüsselkompetenz. Hier darf sich die Statistik nicht zurückziehen, das sage ich gerade aus der Perspektive meiner Tätigkeit am IAB.

In den Anwendungsdisziplinen gibt es große Unterschiede in der Ausrichtung der im weitesten Sinn der Statistik zuzurechnenden Professuren. In der Volkwirtschaftslehre etwa ist es gängige Praxis, dass die Professuren einen Beitrag zur Methodenentwicklung leisten, so dass sich Anwendungsdisziplin und Methodenentwicklung befruchten. Das ist jedoch nicht überall der Standard. Wenn methodische statistische Grundlagenforschung nicht innerhalb einer Anwendungsdisziplin stattfindet, dann fehlt eine entscheidende Triebfeder, um den statistischen Instrumentenkasten, der für die jeweilige Disziplin wichtig ist, weiterzuentwickeln.

\section{Und die Datenakquise ...}

... darf natürlich ebenfalls nicht vernachlässigt werden. Eine große Stärke in der Methodenausbildung im Bereich der quantitativen Sozialwissenschaften sehe ich darin, dass die Studierenden wesentlich intensiver als in den Wirtschaftswissenschaften Kenntnisse zu den Themen Datenentstehung und Datenerhebung, Stichprobenplanung und Survey-Statistik erwerben. Vergleichbare Kenntnisse zu diesen Themen - oder praktisches Wissen zu den Grundlagen der Wirtschafts- und Sozialstatistik (früher ein Standardthema in der Ausbildung zur empirischen Wirtschaftsforschung, siehe das Lehrbuch von Hujer und Cremer 1978) - vermisse ich gelegentlich bei Studierenden aus den Wirtschaftswissenschaften, die ansonsten eine ausgezeichnete Ausbildung in theoretischer und/oder angewandter Ökonometrie haben. Aber als Ökonom weiß man, dass man nicht alles haben kann.

Von der Ausbildung in das wahre Leben. Ich habe mehrfach die These vertreten (siehe etwa Grohmann-Vorlesung, Krämer 2016), dass unsere Wirtschaft jährlich netto mehrere 100.000 Arbeitskräfte aus dem Ausland braucht. Was sagen Sie als Experte dazu?

Rein quantitativ stimme ich der These im Wesentlichen zu. Ohne Zuwanderung können wir den Arbeitskräftebedarf unserer Wirtschaft nicht voll decken - und ohne Zuwanderung ergäben sich auch dramatische Folgen für unser System der sozialen Sicherung. Bei einer schrumpfenden Wirtschaft infolge von zunehmender Arbeitskräfteknappheit würden die Steuereinnahmen zurückgehen und die Beitragszahlungen fehlen, die wir insbesondere für die Rentenversicherung benötigen. Die Problematik ist schon in den Jahren 2020 und 2021 deutlich sichtbar geworden. Im beiden Jahren sank alterungsbedingt die Zahl der Personen, die dem Arbeitsmarkt insgesamt zur Verfügung stehen, jährlich um 300.000 bis 350.000 Personen. Die Erwerbsbeteiligung im Inland, bspw. über die weiter zunehmende Erwerbstätigkeit von Frauen, wuchs nach Rückgängen am Anfang der Covid-19-Pandemie im Jahr 2021 um gut 100.000 Personen - und damit langsamer als im letzten Jahrzehnt. Und die Immigration nach Deutschland ist in der Corona-Krise deutlich zurückgegangen, so dass dem Arbeitsmarkt in Deutschland zwei Jahre in Folge weniger Arbeitskräfte 
zur Verfügung standen. Schon im zweiten Halbjahr 2021 waren in einigen Bereichen wieder Arbeitskräfteengpässe zu erkennen.

Der Demographie-Effekt dürfte im nächsten Jahrzehnt in jedem Jahr in der gleichen Größenordnung liegen. In der Summe müssten daher 300.000 bis 400.000 Personen pro Jahr mehr zuwandern als unser Land verlassen, um die Zahl der dem Arbeitsmarkt zur Verfügung stehenden Personen konstant zu halten. In Folge der Covid-19-Pandemie ist die Zuwanderung allerdings kurzfristig zurückgegangen und die Zuwanderungspotenziale aus anderen EU-Ländern sind geringer als vor einigen Jahren, da auch dort vergleichbare demographische Effekte wirken.

Sind wir auf dem richtigen Weg? Die Politik hat richtigerweise mit dem Berufsanerkennungsgesetz und dem Fachkräfteeinwanderungsgesetz die Hürden für die Einwanderung von vor allem benötigten Fachkräften gesenkt und unterstützende Maßnahmen auf den Weg gebracht. Das IAB wird evaluieren, ob das Fachkräfteeinwanderungsgesetz die intendierten Effekte nach Ende der Corona-Krise zeigen wird.

Sie waren lange Jahre ein wichtiger Gutachter für die Deutsche Forschungsgemeinschaft. Wie beurteilen Sie den Stellenwert dieser Organisation im deutschen Wissenschaftsgefïge?

Der ist sehr hoch einzuschätzen. Ich wurde zweimal in das Fachkollegium Wirtschaftswissenschaften für das Gebiet Statistik und Ökonometrie gewählt, hatte also die Ehre, insgesamt acht Jahre in diesem Gremium mitzuarbeiten, darunter zwei Jahre als stellvertretender Vorsitzender und zwei Jahre als Vorsitzender. Für meine zweite Amtszeit war ich sowohl vom Verein für Socialpolitik wie auch von der Deutschen Statistischen Gesellschaft (DStatG) als Kandidat vorgeschlagen worden. Hierüber habe ich mich besonders gefreut - und danke der DStatG für das mir entgegengebrachte Vertrauen. Besonders spannend und gleichzeitig herausfordernd war es dabei für mich, angemessen die Fachspezifika und Fachkulturen in einem Gremium zu berücksichtigen, in dem BWL, VWL, Wirtschafts- und Sozialgeschichte und eben Statistik und Ökonometrie zusammen vertreten sind. Die Vertraulichkeit der Beratungen verbietet mir, auf Einzelheiten einzugehen, aber die unterschiedlichen Fachkulturen führten zu teilweise leicht unterschiedlichen Kriterien für die Förderentscheidungen und zu leicht unterschiedlichen Einschätzungen der Sinnhaftigkeit einzelner Förderinstrumente. Für das Gremium ist es daher wichtig, ein gemeinsames Verständnis für angemessene und faire Förderentscheidungen zu entwickeln, die den Anträgen in ihrer Heterogenität und den eingeholten Gutachten gerecht werden und die die Wirtschaftswissenschaften insgesamt voranbringen. Die angemessenen Maßstäbe für Anträge an der Schnittstelle verschiedener Fächer - sei es an der Schnittstelle Statistik und Mathematik oder an der Schnittstelle zwischen Wirtschaftsgeschichte und Geschichtswissenschaft - stellten hierbei eine Herausforderung dar, die das Fachkollegium nach meiner Einschätzung in meinen zwei Amtsperioden gut gemeistert hat. Dabei haben wir uns gelegentlich und nach ausführlicher Diskussion auch schon mal über den Mainstream in VWL und BWL hinweggesetzt. (Abb. 4). 
Abb. 4 Bernd Fitzenberger (rechts) beim internationalen BIBB-IAB-ZEW-Workshop „Tasks III: Changing tasks Consequences for inequality “ am 26.-28. Mai 2014 in der Zentrale der Bundesagentur für Arbeit. Links Joachim Möller

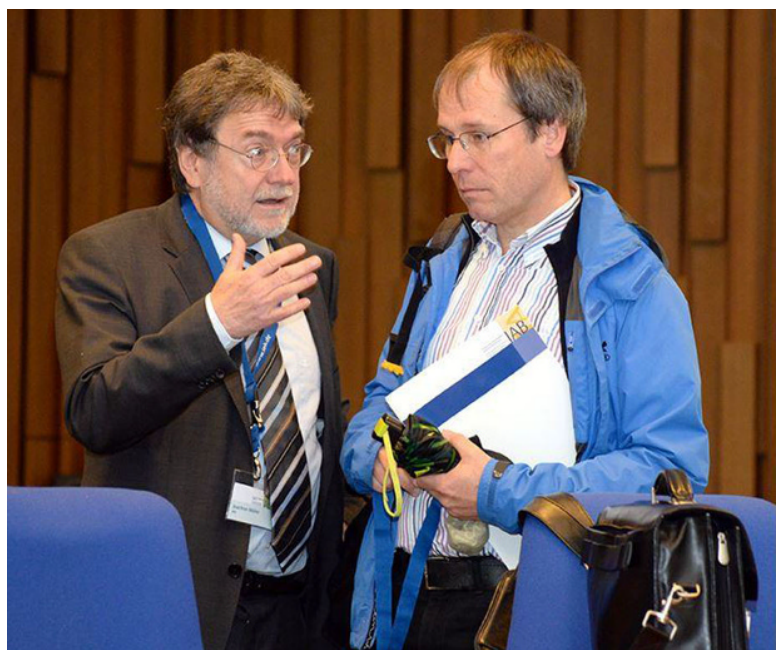

Irgendwelche Empfehlungen für Ihre Nachfolger?

Anders als bei den von der EU vergebenen ERC grants ist bei der DFG auch die Förderung von Nachwuchswissenschaftlerinnen und -wissenschaftlern ganz am Anfang ihrer Karriere ein wichtiges Ziel. Die Bedeutung und Konzeption dieser Nachwuchsförderprogramme ist aber dem Nachwuchs in Deutschland noch nicht hinreichend bekannt. Deshalb finde ich es gut und wichtig, dass die DFG auf den Tagungen der Fachgesellschaften hierüber informiert - hieran habe ich mich immer gerne beteiligt - und der Nachwuchs die Beratungsleistung der DFG vor der Antragstellung nutzt. Das könnte man noch weiter intensivieren.

Von der DFG zur Forschung und zu den deutschen Universitäten allgemein. In einem kürzlichen Interview mit Gerd Hansen (Krämer 2022) haben sowohl ich als auch der Interviewte das geistige Klima an deutschen Universitäten heutzutage kritisiert. Wie sehen Sie das denn?

Mit dem Begriff ,geistiges Klima“ tue ich mir schwer. Speziell bei dem Begriff „Geist“ im Zusammenhang mit Universitäten erinnere ich mich an ein für mich unvergessliches Radio-Interview mit Walter Jens. Walter Jens galt bekanntermaßen als streitbarer Geist und scharfzüngiger Denker, dessen Ansichten ich nicht immer teilte, aber den ich sehr schätzte. Auf die Frage hin, wie es passieren konnte, dass er im kleinen Tübingen lehre und dort geblieben sei, antwortete Walter Jens u.a., dass ihn gleich als er nach Tübingen gekommen sei, der „Geischt“ [schwäbisch für Geist] dort angesprochen habe. Ich habe das so verstanden, dass er das ,geistige Klima“, sprich die Form und die Inhalte des akademischen Austausches, die Kommunikation in Universität und Stadtgesellschaft und auch die Streitkultur im Tübingen der 1950er-Jahre geschätzt hat.

Lebt der von Walter Jens geschätzte Geist in Form rhetorisch geschliffener aber akademisch gehaltvoller und respektvoller Kommunikation und Diskussion, die hart in der Sache ist, an den deutschen Universitäten heute fort? Ich halte dies für ein 
schönes Ideal, eine schöne Utopie, und schätze sehr, wenn ich dieses Ideal in Realität erlebe - wofür es an deutschen Universitäten immer wieder schöne Beispiele gibt. Aber es wäre ein zu hoher, elitärer Anspruch, um die Realität an deutschen Universitäten daran zu messen, denn die Universitäten sind natürlich Teil der Gesellschaft, deren Verhaltensmuster sich nicht unbedingt an humanistischen Bildungsidealen orientiert. Die Universitäten spiegeln somit auch negative Entwicklungen in der Gesellschaft wider. Im großen Ganzen garantieren sie in der öffentlichen Diskussion und in Auseinandersetzung über unterschiedliche Meinungen ein hohes Maß an Freiheit und kritischem Diskurs, auch wenn dies nicht völlig dem skizzierten Ideal entspricht. Ich schätze die Offenheit und das Engagement, mit denen beispielsweise Studierende der Wirtschaftswissenschaften alternative Sichtweisen in unser Lehrprogramm einbringen oder mit der sie sich für politische Forderungen einsetzen, selbst wenn ich diese nicht teile. Dies ist an deutschen Universitäten gut möglich, ohne dass der oder die Betreffende Nachteile befürchten muss.

Schockierend dagegen - und meinem Idealbild des geistigen Klimas an einer Universität widersprechend - ist beleidigende oder respektlose Kommunikation, wie sie teilweise in anonymer Form in sozialen Medien oder in Kommentarspalten im Internet vorkommt. Dazu zähle ich anonyme, beleidigende Kritik an Lehrenden, wie ich sie leider viel zu oft auf Bewertungsplattformen, Onlineforen oder in anonymen Lehrevaluationen für meine Person oder Kolleginnen und Kollegen gelesen habe. Auch sexistische Äußerungen über weibliche Lehrende sind hier zu beklagen. Keine Frage: Berechtigte Kritik und kritische Diskussionen müssen sein - ich bin offen dafür und bewundere Studierende und die Vertreterinnen und Vertreter anderer Statusgruppen an der Universität, wenn sie offen Missstände ansprechen, ihre Einschätzungen einbringen und sich an Entscheidungsfindungsprozessen beteiligen. Aber beleidigende und entwürdigende Kommunikation in Anonymität ist immer unangemessen und einer Universität unwürdig - und ich vermisse einen höheren Schutz der Adressaten dieser Kommunikation an den Universitäten, aber auch in der Gesellschaft insgesamt.

Da sind wir ja schon zwei - gibt es etwas, das Sie dem neuen Vorsitzenden der DStatG mit auf den Weg geben könnten?

Ich möchte meine Antwort als zwei Einschätzungen verstanden wissen, die ich zur Diskussion stelle. Erstens, der aktuelle Hype im Bereich Data Science, Maschinellem Lernen, Künstliche Intelligenz in Verbindung mit der Verfügbarkeit von neuen Datentypen ist eine große Chance, das Fach Statistik zu stärken. Bei Anwendungen dieser Methoden in den Wirtschaftswissenschaften ist eine dezidiert statistische und ökonometrische Perspektive sinnvoll und wichtig. Zugespitzt formuliert heißt das: Die Frage der angemessenen statistischen Inferenz, Messfehlerprobleme oder Selektionseffekte, um nur drei Beispiele zu nennen, verschwinden mit den neuen Methoden nicht von selbst.

Zweitens, die DStatG stellt sich vielen wichtigen Aufgaben - das ist großartig, birgt aber besondere Herausforderungen. Lassen Sie mich zwei davon nennen. Amtliche Statistik und Wissenschaft/Lehre an den Universitäten kontinuierlich zusammenzubringen ist sehr wichtig, gerade weil der Stellenwert der Vermittlung von Kenntnissen in der Wirtschafts- und Sozialstatistik an den Universitäten höher sein 
sollte und weil die amtliche Statistik von dem Austausch mit der Hochschulforschung profitiert. Eine Herausforderung entsteht daraus, dass die amtliche Statistik größtenteils, wenn auch nicht ausschließlich (Beispiel: Eurostat), national aufgestellt ist, während die Methodenforschung als Grundlagenforschung durch und durch international ist.

Und Statistik ist in vielen Anwendungsdisziplinen vertreten, von denen die DStatG nur einige repräsentiert. Die Herausforderung besteht darin, den Austausch im Fach Statistik über die Anwendungsdisziplinen hinweg zu ermöglichen und zu organisieren. Für beide Herausforderungen hat die DStatG funktionierende Lösungen gefunden, die jedoch regelmäßig einer Überprüfung und Anpassung an sich verändernde Rahmenbedingungen bedürfen.

Das ist ein schönes Schlusswort. Ich danke Ihnen für das Gespräch.

\section{Literatur}

Fitzenberger B (1998) The moving blocks bootstrap and robust inference in linear least squares and quantile regressions. J Econom 82(2):235-287

Fitzenberger B (1999) Wages and employment across skill groups: an analysis for west Germany. Physica, Heidelberg

Fitzenberger B, Hujer R, MaCurdy TE, Schnabel R (2001) Testing for uniform wage trends in west Germany: a cohort analysis using quantile regressions for censored data. Empir Econ 26(1):41-86

Fitzenberger B, Schnabel R, Wunderlich G (2004) The gender gap in labor market participation and employment: a cohort analysis for west Germany. J Popul Econ 17(1):83-116

Hujer R, Cremer R (1978) Methoden der empirischen Wirtschaftsforschung: Verfahren zur Diagnose und Prognose makroökonomischer Prozesse. Vahlen,

Krämer W (2016) Die demografische Zeitbombe: Ursachen und Folgen der Kinderlosigkeit (Heinz-Grohmann-Vorlesung). Wirtsch Sozialstat Arch 10:305-323

Krämer W (2022) Interview mit Gerd Hansen. Wirtsch Sozialstat Arch 15:293-302

Ronning G (1991) Mikroökonometrie. Springer, Berlin Heidelberg

Hinweis des Verlags Der Verlag bleibt in Hinblick auf geografische Zuordnungen und Gebietsbezeichnungen in veröffentlichten Karten und Institutsadressen neutral. 